\title{
Single-balloon enteroscopy efficacy and degree of concordance with noninvasive evaluation of small bowel
}

\section{(우요 $\odot$}

\author{
Authors \\ Margarida Marques, João Santos-Antunes, Rosa Coelho, Hélder \\ Cardoso, Filipe Vilas Boas, Armando Ribeiro, Guilherme Macedo \\ Institutions \\ Gastroenterology Department, Centro Hospitalar São João, Porto \\ (Porto) \\ submitted 21.12.2015 \\ accepted after revision $\quad 24.10 .2016$ \\ Bibliography \\ DOI http://dx.doi.org/10.1055/s-0042-121415 | \\ Endoscopy International Open 2017; 05: E96-E102 \\ (c) Georg Thieme Verlag KG Stuttgart · New York \\ ISSN 2364-3722 \\ Corresponding author \\ Margarida Marques, Centro Hospitalar São João, Porto, \\ Departement of Gastroenterology, Alameda Professor Hernâni \\ Monteiro Porto, Porto 4200-319, Portugal \\ Phone: 225512100 \\ Fax: 225025766 \\ marquesm@ufp.edu.pt
}

\begin{abstract}
Background and study aims Clinical impact of single-balloon enteroscopy (SBE) is fairly known, as well as its diagnostic yield comparing with other small bowel gastrointestinal investigations. This study represents a contribution to better understand it and is designed to

evaluate SBE efficacy and degree of concordance with previous evaluation of small bowel.

Patients and methods This is a single-center retrospective study of patients that underwent SBE with suspected small bowel disease based on non-invasive imaging. Demographic, clinical, procedural and outcome data were collected for analysis. Agreement beyond positive findings was evaluated using $\mathrm{k}$-coefficient.

Results A total of 197 SBEs were performed in 168 patients; mainly men $(64.3 \%)$ with mean age $53.3 \pm 17.6$ years. Most SBEs ( $86.3 \%)$ performed were preceded by a noninvasive evaluation: in $61.4 \%$ (n $=119$ ) of cases, capsule enteroscopy (CE) was performed, in $18.8 \%$ $(n=37)$, computed tomography was performed, and in $6.1 \%(n=12)$ magnetic resonance enterography was performed. Fourty-three patients $(25.6 \%)$ underwent endoscopic treatments, mainly: argon plasma coagulation in angioectasias (53.4\%) and polypectomy (34.9\%). The most common diagnoses made with SBE were findings consistent with inflammatory small bowel disease $(21.8 \%)$ and vascular lesions (14.2\%). The diagnostic yield of SBE was of $69 \%$, confirming the suspicion of small bowel disease. The degree of concordance between CE and SBE for positive findings was substantial, $\mathrm{K}$ coefficient $=0.635(P<0.001)$. However, the degree of concordance between imaging examinations (CT or MR) and SBE was only moderate, $\mathrm{k}$-coefficient $=0.410(P<0.001)$. SBE had an immediate effect in $20 \%$ of patients, changing diagnostic approaches, medical and surgical treatments.

Conclusions Our study supports the idea that for suspected small bowel disease, CE and SBE have an overall good degree of concordance for all the diagnostics included.
\end{abstract}

\section{Introduction}

The small bowel has long been considered a black box for endoscopists because of its length and the presence of multiple complex loops. For several years, mucosal visualization of the small bowel was limited to the reach of push enteroscopy and ileocolonoscopy, with the exception of intraoperative enteroscopy. Development of capsule endoscopy (CE) and device-assisted enteroscopy (DAE) has permitted observation of the entire small bowel.

The first deep-enteroscopy technique, developed in 2001, was double-balloon enteroscopy (DBE) [1]. In 2008, a novel, simplified system of single-balloon enteroscopy (SBE) was introduced, using 1 instead of 2 balloons. Instead of the endoscope tip balloon in DBE, SBE involves angling the endoscope tip or power suction to achieve stable positioning in the small bowel [2]. The main therapeutic indications for DAE include need for treatment of small intestinal lesions found on other gastrointestinal investigations, such as CE or radiologic examinations [3].

The primary aim of the current study was to retrospectively evaluate the efficacy of SBE, in terms of diagnostic and therapeutic yield in patients with suspected small bowel disease, and to evaluate the degree of concordance between SBE and other previous investigations, such as $C E$ or radiologic examinations. Secondary aims were to assess SBE safety, the procedure success and insertion depth, and outcome of patients submitted to SBE. 


\section{Patients and methods}

A retrospectively study was performed at a single Portuguese center. That institution is a tertiary hospital and a referral center for small bowel diseases. Each patient gave verbal and signed informed consent for the procedures and all rules of local ethics committee were followed, preserving patient identity and confidentiality.

We evaluated patients with suspected small bowel diseases, from June 2010 to December 2014. The patients were mainly being investigated for suspected obscure gastrointestinal bleeding, suspected small bowel tumors, and Crohn's disease evaluation. All patients had negative upper and lower gastrointestinal endoscopy before referral. Moreover, standard endoscopy was performed twice on patients with suspected obscure gastrointestinal bleeding, as is recommended.

All patients included had been subjected to previous noninvasive evaluation of small bowel with $\mathrm{CE}$, computed tomography $(C T)$ or magnetic resonance (MR) enterography, which revealed a suspicion lesion in the middle gut.

The SBE system used comprised the SIF-Q160 endoscope (Olympus Optical Company, Ltd, Tokyo, Japan), which has a working length of $200 \mathrm{~cm}$, a $9.8-\mathrm{mm}$ diameter, and contains a 2.8-mm diameter working channel, the Balloon Control Unit OBCU, and the ST-SB1 single-use splinting tube (overtube), which measures $132 \mathrm{~cm}$ in length and has an outer diameter of $13.2 \mathrm{~mm}$.

The splinting tube's smooth glide, hydrophilic coating is activated with the simple addition of $30 \mathrm{~mL}$ of water. The SBE is controlled by repeatedly inflating and deflating a single balloon, attached to the distal end of the splinting tube, via the remote balloon controller.

To improve maneuverability of insertion, the SIF-Q160 features a distal end diameter of just $9.2 \mathrm{~mm}$ while maintaining high-resolution image quality. By optimizing both the distal end length and bending section radius, the SIF-Q160 extensive angulation capability allows acute turns in the small intestine, which allows smoother insertion. The ST-SB1 single-use splinting tubes used as over-tubes are made from silicone rubber to eliminate risk of latex allergy [3].

The starting insertion route of SBE (oral or anal) was chosen as per clinical judgment according to clinical presentation or to the probable location of the suspected lesions on the basis of the findings of previous noninvasive small bowel investigation. This means that the antegrade route typically was used for lesions located within the proximal two-thirds of the small bowel, whereas the retrograde route was used for lesions in the distal one-third, based on capsule endoscopy transit times.

Technical success of small bowel examination was defined as scope passage beyond the ligament of Treitz by the oral approach or stable intubation of the terminal ileum to at least $20 \mathrm{~cm}$ beyond the ileocecal valve.

The length of small bowel segments explored was measured according to the method described by May et al [4].

Most SBE procedures were done in an outpatient setting, without fluoroscopic control. All patients received monitored anesthesia care with propofol. Carbon dioxide was used as in- sufflation gas during enteroscopy. Data were collected and entered into a dedicated structured database.

\section{Statistical analysis}

Categorical variables were described as absolute ( $n$ ) and relative frequencies (\%). Mean and standard deviation or median and percentiles were used for continuous variables as appropriate. Cohen's kappa statistic test was used to evaluate concordance between the diagnostic tests. The significance level used was 0.05 . Statistical analysis was performed using the software Statistical Package for the Social Sciences v.22.0.

\section{Results}

During the study period, a total of 197 SBE procedures were performed, 122 by the oral route, 75 by the anal route, with 6 done by routes, in 168 patients (108 men) with a mean age of $53.3 \pm 17.6$. Demographic and clinical characteristics of patients included are represented in $>$ Table 1 .

Some patients $(n=19)$ were subjected to more than 1 SBE, either by the same or different route. All patients had undergone a previous noninvasive small bowel study, which led to the indication for SBE. Patients who had undergone more than one SBE did not have a previous noninvasive study repeated. - Table 1 reports indications for all SBE included in this study.

The main indications for SBE were anemia/obscure gastrointestinal bleeding or overt gastrointestinal bleeding of small bowel (31.4\%) and established Crohn's disease (24\%). SBE was performed under anesthesia care with propofol and the mean procedure time was $74 \pm 39$ min for the oral route and $81 \pm 47 \mathrm{~min}$ for the anal route. Mean insertion depth for SBE was $232 \pm 87 \mathrm{~cm}$ beyond the ligament of Treitz (range 20$400 \mathrm{~cm}$ ) and $91 \pm 50 \mathrm{~cm}$ beyond the ileocecal valve (range $15-170 \mathrm{~cm}$ ). There were no failures on the intention to perform SBE in either oral or anal procedures. Four of 6 patients, who were submitted to oral and anal route, had a complete enteroscopy. These patients started with anterograde SBE and the deepest point of insertion was marked with black ink. Afterwards patients were rescheduled another day for retrograde SBE and in 4 patients, the black marker was reached.

Prior to SBE, noninvasive evaluation of small bowel was performed by CE in 119 procedures, CT in 37 and by MR in 12. In 29 SBEs, an imaging study was not repeated before enteroscopy, most likely because the diagnosis was known and/or clinical suspicion was strong enough.

Major diagnoses were findings consistent with inflammatory small bowel disease (20.9\%), vascular lesions (20.9\%), with or without active bleeding, and polyps (15.7\%). The diagnostic yield of SBE for inflammatory disease was quite high considering vascular lesions because our department is a reference center for inflammatory bowel diseases, with a large number of patients. The overall yield of SBE for diagnostic findings was of $69 \%$, however, when only clinically relevant findings were considered, the yield was slightly lower, 62\%. In 61 SBEs (31\%) performed, small bowel mucosa was normal, excluding the diagnosis in those patients or in some admitting that the suspected area was not reached. Total enteroscopy was not attempted in 
- Table 1 Demographic, clinical characteristics and indications for SBE of patients enrolled in the study $(n=168)$.

\begin{tabular}{|c|c|}
\hline Variable & n (\%) \\
\hline Male gender, n (\%) & $108(64.3 \%)$ \\
\hline Median age (IQR, years) & $53(12-85)$ \\
\hline Previous abdominal surgery, $\mathrm{n}(\%)$ & $29(17.3 \%)$ \\
\hline AINE's in the last 6 months, $n(\%)$ & $7(4.2 \%)$ \\
\hline Anticoagulation therapy, $\mathrm{n}(\%)$ & $14(8.3 \%)$ \\
\hline \multicolumn{2}{|l|}{ Previous small bowel imaging } \\
\hline - CE & $119(70.8 \%)$ \\
\hline - $\mathrm{CT}$ & $37(22.1 \%)$ \\
\hline - $\mathrm{MR}$ & $12(7.1 \%)$ \\
\hline \multicolumn{2}{|l|}{ Indications } \\
\hline - GI bleeding of middle gut & $32(16.2)$ \\
\hline - Anemia & $30(15.2)$ \\
\hline - Crohn's disease evaluation & $47(23.8)$ \\
\hline - Polyposis & $20(10.2)$ \\
\hline - Polyps & $16(8.1)$ \\
\hline - Diagnosis/surveillance of lymphoma & $15(7.6)$ \\
\hline - Chronic diarrhea & $11(5.1)$ \\
\hline - Suspected tumor & $10(5.1)$ \\
\hline - Surveillance after surgery & $8(4.1)$ \\
\hline - Celiac disease & $4(2.0)$ \\
\hline - Foreign body & $3(1.5)$ \\
\hline - Other & $1(0.5)$ \\
\hline - Total & $197(100)$ \\
\hline $\begin{array}{l}\mathrm{CE} \text {, capsule endoscopy; } \mathrm{CT} \text {, computed tomo } \\
\text { nance imaging }\end{array}$ & magnetic reso- \\
\hline
\end{tabular}

these patients for several reasons: most had clinical improvement in their medical condition; some patients refused to submit to a new procedure; and in a few patients the diagnosis was assumed after the initial enteroscopy.

In relation to therapeutic yield, 43 patients (25.6\%) underwent endoscopic treatments. Argon plasma coagulation (APC) was performed in 23 patients (53.4\%) to treat angioectasias, half of whom had ongoing bleeding. Previously to delivery $A P C$, injection of mucosa with saline was performed, reducing the risk of small bowel injury. APC was effective in hemostasis and eradication of those vascular anomalies. Polypectomy was performed in 15 patients (34.9\%), most of whom had Peutz-Jeghers syndrome and familial adenomatous polyposis. The injection-assisted (or "inject-and-cut") technique of endoscopic mucosal resection (EMR) was used in sessile lesions/polyps: submucosal injection of saline followed by application of snare cautery for lesion resection was performed in each polypectomy. The injection was delivered via a standard injection needle.
Typically, $5 \mathrm{~mL}$ to $15 \mathrm{~mL}$ of saline were injected beneath the lesion. The addition of indigo carmine assisted in identification of deep and lateral resection margins in larger lesions. In large pedunculated polyps, such as in Peutz-Jeghres polyposis, a hemoclip was applied to the pedicle, previously polypectomy, to prevent bleeding. Additional therapeutic interventions are shown at $>$ Table 1 (Supplementary material).

No patient developed major complications related to SBE, during or after the procedure, which was well tolerated in all cases. One patient $(0.50 \%)$ had a drop in oxygen saturation during SBE, which resulted in temporary suspension of the procedure, but afterwards it was completed. We recorded 2 patients who needed medication for mild abdominal pain after SBE. None of the patients who underwent SBE in the outpatient clinic were admitted because of complications related to the procedure.

Most SBEs (86.3\%) included in this study were preceded by noninvasive evaluation of small bowel: $61.4 \%(n=119)$ performed CE, 18.8\% $(n=37)$ CT and $6.1 \%(n=12)$ MR. Because all patients submitted to CE underwent bowel preparation, the quality of acquired images was good. The mean transit time of the CE through the small bowel was $287.3 \pm 93.4 \mathrm{~min}$. In $17 \mathrm{pa}$ tients CE was not completed, i. e. did not reach the cecum. Patients who had previous abdominal surgery and/or symptoms of intestinal obstruction with risk of retained capsule were subjected to patency capsule testing before CE.

Forty-nine SBEs (24.9\%) included were preceded by 1 of 2 radiologic methods: CT $(n=37,18.8 \%)$ or $M R(n=12,6.1 \%)$. These were performed in patients who were not candidates for $\mathrm{CE}$, or for other reasons were subjected to one of the methods and small bowel disease was suspected. All patients were given intravenous and oral contrast.

To calculate the concordance between imaging modalities the authors included all diagnoses evaluated. Overall, the degree of concordance between SBE and CE for positive findings was substantial, with a calculated $\mathrm{k}$-coefficient of $0.635(P<$ $0.001)$. The degree of concordance between procedures according to type of lesion is presented in $\mathbf{T}$ Table $\mathbf{2}$.

In spite of the overall concordance described above, we also calculated the concordance between SBE and CE, dividing patients into subgroups according to the main indication for SBE: obscure bleeding or CD evaluation. In patients subjected to investigation for obscure bleeding, the concordance between SBE and CE was substantial, with a calculated $\mathrm{k}$-coefficient $=0.601$. Of all CE procedures with positive findings, SBE managed to identify the lesion in $68 \%$ of patients. Considering patients submitted to investigation for $C D$, the concordance was only moderate with a calculated $\mathrm{k}$-coefficient of 0.417 .

Overall, degree of concordance between SBE and imaging examinations for positive findings was only moderate, with a calculated $\mathrm{k}$-coefficient of $0.410(P<0.001)$. Degree of concordance between procedures according to type of lesion is presented in $>$ Table 3 and $>$ Table 4 . Of all CT procedures with positive findings, SBE managed to identify the lesion in $40 \%$ of patients. We do not have sufficient patients to compare all 3 modalities. 
- Table 2 Type of lesions detected with CE and SBE.

\begin{tabular}{|c|c|c|c|c|c|c|c|c|}
\hline & \multicolumn{7}{|l|}{ SBE } \\
\hline & & $\begin{array}{l}\text { No } \\
\text { lesion }\end{array}$ & $\begin{array}{l}\text { Active bleeding/clots } \\
\text { with unknown origin }\end{array}$ & $\begin{array}{l}\text { Vascular } \\
\text { lesions }\end{array}$ & $\begin{array}{l}\text { Polyps/ } \\
\text { tumors }\end{array}$ & $\begin{array}{l}\text { Inflammatory } \\
\text { lesions }\end{array}$ & Melanose & Total \\
\hline \multirow{8}{*}{ CE } & No lesion & 10 & 0 & 0 & 1 & 1 & 0 & 12 \\
\hline & $\begin{array}{l}\text { Active bleeding/clots } \\
\text { with unknown origin }\end{array}$ & 4 & 1 & 0 & 0 & 0 & 0 & 5 \\
\hline & Vascular lesions & 7 & 0 & 25 & 0 & 1 & 0 & 33 \\
\hline & Polyps/tumors & 7 & 0 & 0 & 20 & 1 & 0 & 28 \\
\hline & Inflammatory lesions & 11 & 0 & 0 & 1 & 27 & 0 & 40 \\
\hline & Flat mucosa & 2 & 0 & 0 & 0 & 0 & 0 & 2 \\
\hline & Melanose & 0 & 0 & 0 & 0 & 0 & 1 & 1 \\
\hline & Total & 41 & 1 & 25 & 22 & 31 & 1 & 122 \\
\hline
\end{tabular}

- Table 3 Type of lesions detected with CT and SBE.

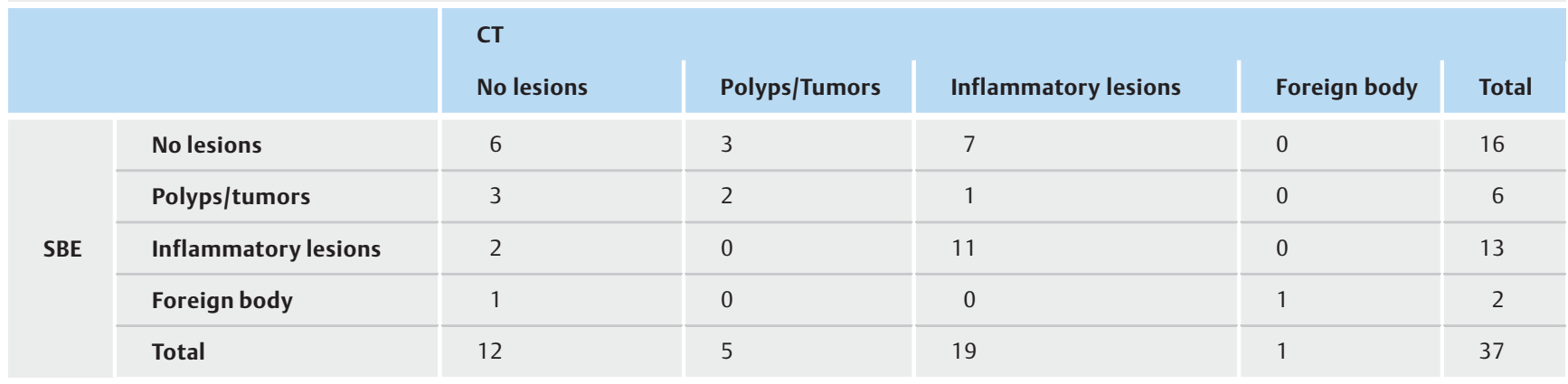

- Table 4 Type of lesions detected with MR and SBE.

\begin{tabular}{|c|c|c|c|c|}
\hline & & \multicolumn{3}{|l|}{ MR } \\
\hline & & No lesions & Inflammatory lesions & Total \\
\hline \multirow{4}{*}{ SBE } & No lesions & 5 & 2 & 7 \\
\hline & Polyps/Tumors & 3 & 0 & 3 \\
\hline & Inflammatory lesions & 0 & 2 & 2 \\
\hline & Total & 8 & 4 & 12 \\
\hline
\end{tabular}

SBE had an immediate effect in $20 \%(n=35)$ of patients included, enabling introduction of endoscopic procedures, altering decisions about medical and/or surgical treatments, and modifying outcomes. Most patients with bleeding vascular lesions were subjected to more than 1 session of APC, with hemoglobin stability thereafter. Moreover, managment was altered in 31 of 168 CD patients (18.5\%), which makes SBE an important technique in this setting, in spite of previous noninvasive small bowel evaluation.

\section{Discussion}

Diagnostics for small bowel diseases have evolved since the advent of both CE and DAE. Enteroscopy typically refers to more extensive endoscopic examination of the small intestine, ex- tending into the jejunum and/or ileum. Diagnostic evaluation of the small bowel can be performed by noninvasive imaging (CT or MR enterography) or by wireless capsule endoscopy. Whereas these modalities currently lack therapeutic ability, they often precede and serve to guide and direct therapy via enteroscopy [5]. In our study, most patients undergoing SBE had a previous noninvasive evaluation of small bowel: in 29 enteroscopies, noninvasive small bowel evaluation was not performed as the diagnosis was known or clinical suspicion was strong. The most common indication for all types of enteroscopy is diagnosis and/or therapy of obscure overt or occult intestinal bleeding, i.e., bleeding without an etiology found on standard upper endoscopy and colonoscopy with terminal ileoscopy. Other indications include evaluation of imaging abnormalities that raise concern for small bowel Crohn's disease, stric- 
tures, ulcers, celiac disease, malabsorption, polyps, masses, lymphoma, and other infiltrative diseases [5]. In the current study, and in accord with the literature, our leading indication was bleeding of the middle gut. The second most important indication was Crohn's disease because our institution is a referral center for inflammatory bowel diseases.

Reported diagnostic yields of SBE have ranged from $41 \%$ to $65 \%$ and therapeutic yields from $7 \%$ to $50 \%$. The reported range for depth of insertion is $133 \mathrm{~cm}$ to $270 \mathrm{~cm}$ for antegrade examinations and $73 \mathrm{~cm}$ to $199 \mathrm{~cm}$ for retrograde examinations [5]. Our diagnostic yield for SBE was $62 \%$, with a therapeutic yield of $25.6 \%$. Repeated hemostasis is often required, especially in patients with vascular lesions and comorbidities such as renal or cardiac disease [6]. These data show that SBE, despite being an invasive procedure, appears to be a suitable endoscopic method required both for more precise diagnosis of suspected small bowel diseases and determination of the most appropriate treatment.

Overall, enteroscopy appears to be very safe. Adverse events (Aes) reported with SBE are also rare and include abdominal pain, fever, mucosal tears, pancreatitis, and perforation [5]. Major complications during SBE occur in fewer than $1 \%$ of patients, comparable with the DBE rate. Aktas et al reported no complications in the 145 diagnostic SBE procedures; there was only 1 perforation in the 21 therapeutic SBE procedures, which occurred during dilation of a strictured in the distal ileum and was not related to the SBE technique [1]. Despite being a single-center report, our SBE procedures were always performed by the same endoscopists, who had significant expertise, so that no major AEs were recorded.

In patients in whom a complete enteroscopy was tried (via oral and anal routes), the procedures were scheduled on different days because of procedure time and risk of complications.

Indeed, incomplete visualization of the small bowel still represents the major limitation of the SBE procedure [7]. Reported rates of panenteroscopy with DAE are widely variable, ranging from $8 \%$ to $86 \%$ of patients [ $8-12$ ]. In our series complete enteroscopy was achieved in only 4 patients (2\%). A history of previous abdominal surgery is known to significantly impact insertion depth or total enteroscopy rate [13], and in our study almost one-quarter of SBEs were performed in patients with a history of abdominal surgery. Besides, total enteroscopy was attempted in only a small number of patients.

In sixty-one SBEs (31\%) performed, small bowel mucosa was normal and the diagnosis in these patients cannot be excluded becuase it is possible that the suspicious area was not reached. Total enteroscopy was not attempted in these patients for several reasons: most had clinical improvement in their medical condition; some patients refused to submit to a new procedure; and in a few patients the diagnosis was assumed after the initial enteroscopy.

Our study highlights the usefulness of the combination of noninvasive imaging and SBE for diagnosis of small bowel disease. After disease detection or in emergent cases, SBE should be selected. In this study, SBE proved to be a useful and safe procedure with a high clinical impact (both diagnostic and therapeutic) on management of suspected small bowel diseases.
The diagnostic yield of SBE performed after a previously positive $C E$ is $79.5 \%$, notably higher than the overall diagnostic yield of $62 \%$ for SBE included in this study. A diagnosis suspected with previous small bowel investigations (CE, CT or MR) was confirmed in $57 \%$ of patients in whom SBE was performed.

As for concordance between the findings, we found different results according to the previous method used: It was fair to moderate for CT and MR ( $\mathrm{K}$-coefficient $=0.410)$, but there was substantial agreement between $C E$ and SBE for positive findings ( $\mathrm{k}$-coefficient $=0.635)$. Our data confirm that CE and SBE are complementary, therefore, the optimal strategy seems to be to couple these 2 techniques: CE should be the initial diagnostic test because it is noninvasive, tolerabley, can view the entire small bowel, and can be used to determine the initial route of SBE. Because of its therapeutic capabilities, SBE may be indicated to define and treat lesions identified at capsule endoscopy, or if suspicion for a small bowel lesion is high despite a negative capsule endoscopy, and in patients with active bleeding who show blood or clots in the lumen of the small bowel.

CE findings could provide some useful clues to direct SBE examination in most patients, including: (1) selecting the right patients for SBE; (2) guiding endoscopists to choose the right insertion route for a SBE procedure; (3) providing endoscopists with information about the lesion and making the performance of SBE more focused; and (4) avoiding a second SBE procedure from the opposite route. It is strongly suggested that CE could be used as the initial screening approach in detecting suspected small bowel diseases [14].

For patients with or suspected inflammatory bowel disease who need imaging of small bowel and in whom CE is contraindicated, enterography by CT or MR is a very good option, followed by SBE if needed, prior to therapeutic procedures, such as dilations. However, we evaluated only a small number of patients in whom SBE was performed after a radiologic procedure, so we cannot evaluate concordance in this particular group of patients.

\section{Conclusion}

In conclusion, our study supports the idea that for suspected small bowel diseases, CE and SBE have an overall good degree of concordance for all the diagnostics included. CE, however, has a higher diagnostic yield, considering that some lesions are not seen by SBE. Our data confirm that CE and SBE are complementary and that together they allow physicians to reach more reliable conclusions than either modality alone. Therefore, the optimal strategy seems to be to couple these 2 techniques not only in a sequential but also in a more properly integrated manner.

\section{Competing interests}

\section{None}




\section{References}

[1] Manno M, Riccioni M, Cannizzaro R et al. Diagnostic and therapeutic yield of single-ballon enteroscopy in patients with suspected smallbowel disease: results of the Italian multicenter study. Dig Liver Dis 2013; 45: $211-215$

[2] Wadhwa V, Sethi S, Tewani S et al. A meta-analysis on efficacy and safety: single-balloon vs. double-balloon enteroscopy. Gastroenterology Report 2015; 3: $148-155$

[3] Riccioni ME, Urgesi R, Cianci R et al. Current status of device-assisted enteroscopy: Technical matters, indication, limits and complications. World J Gastrointest Endosc 2012; 4: 453-461

[4] May A, Nachbar L, Schneider M et al. Push-and-pull enteroscopy using the double-balloon technique: method of assessing depth of insertion and training of the enteroscopy technique using the Erlangen Endo-Trainer. Endoscopy 2005; 37: 66 - 70

[5] Chauhan S, Manfredi M, Dayyeh B et al. Enteroscopy. Gastrointest Endosc 2015; 82: 975 - 990

[6] Arakawa D, Ohmiya N, Nakamura M. Outcome after enteroscopy for patients with obscure Gl bleeding: diagnostic comparison between double-balloon endoscopy and videocapsule endoscopy. Gastrointest Endosc 2009; 69: 866 - 874

[7] Marmo R, Rotondano G, Casetti T. Degree of concordance between double-balloon enteroscopy and capsule endoscopy in obscure gas- trointestinal bleeding: a multicenter study. Endoscopy 2009; 41: $587-592$

[8] Yamamoto H, Kita H, Sunada K et al. Clinical outcomes of double-balloon endoscopy for diagnosis and treatment of small intestinal diseases. Clin Gastroenterol Hepatol 2004; 2: 1010 -1016

[9] May A, Nachbar L, Wardak A et al. Double-balloon enteroscopy: preliminary experience in patients with obscure gastrointestinal bleeding or chronic abdominal pain. Endoscopy 2003; 35: 985 - 991

[10] Matsumoto T, Moriyama T, Esaki M et al. Performance of antegrade double-balloon enteroscopy: comparison with push enteroscopy. Gastrointest Endosc 2005; 62: 392 - 398

[11] Monkemuller K, Weigt J, Treiber G et al. Diagnostic and therapeutic impact of double-balloon enteroscopy. Endoscopy 2006; 38: 67-72

[12] Heine GD, Hadithi M, Groenen MJ et al. Double-balloon enteroscopy: indications, diagnostic yield, and complications in a series of $275 \mathrm{pa}-$ tients with suspected small-bowel disease. Endoscopy 2006; 38: 42 48

[13] Khashab M, Helper DJ, Johnson CS et al. Predictors of depth of maximal insertion at double-ballon enteroscopy. Digestive Diseases and Sciences 2010; 55: 1391 - 1395

[14] Li Xi, Dai J, Hong A. Prospective Study on Evaluating the Diagnostic Yield of Video Capsule Endoscopy Followed by Directed Double-Balloon Enteroscopy in Patients with Obscure Gastrointestinal Bleeding. Dig Dis Sci 2010; 55: $1704-1710$

\section{CORRECTION}

The name of João Santos-Antunes was corrected from João Antunes to João Santos-Antunes. 


\section{Supplementary material}

- Table 1 Endoscopic intervention of the SBE performed.

\begin{tabular}{|l|c|c|}
\hline Endoscopic intervention & Frequency (n) & Percent (\%) \\
\hline Biopsy & 77 & 39.1 \\
\hline Small bowel tattoo & 34 & 17.2 \\
\hline Argon plasma coagulation & 23 & 11.7 \\
\hline Polypectomy & 15 & 7.6 \\
\hline Hemoclips & 11 & 5.6 \\
\hline Hemostasis with epinephrine injection & 4 & 2.03 \\
\hline Dilation & 4 & 2.03 \\
\hline Sclerosis & 1 & 0.50 \\
\hline Without endoscopic intervention & 62 & 31.5 \\
\hline
\end{tabular}

